\title{
Natural Hand Gesture Recognition with an Electronic Textile Goniometer
}

\author{
Sang-Ho Han, ${ }^{1}$ Eun-Ji Ahn, ${ }^{1}$ Mun-Ho Ryu, ${ }^{2,3 *}$ and Je-Nam Kim ${ }^{4}$ \\ ${ }^{1}$ Department of Healthcare Engineering, Chonbuk National University, \\ 567 Baekje-daero, Deokjin-gu, Jeonju-si, Jeollabuk-do 561-756, Republic of Korea \\ ${ }^{2}$ Division of Biomedical Engineering, Chonbuk National University, \\ 567 Baekje-daero, Deokjin-gu, Jeonju-si, Jeollabuk-do 561-756, Republic of Korea \\ ${ }^{3}$ Research Center of Healthcare \& Welfare Instrument for the Aged, \\ 567 Baekje-daero, Deokjin-gu, Jeonju-si, Jeollabuk-do 561-756, Republic of Korea \\ ${ }^{4}$ CAMTIC Advanced Mechatronics Technology Institute for Commercialization, \\ 67 Yu-Sang-ro, Deokjin-gu, Jeonju-si, Jeollabuk-do 561-844, Republic of Korea
}

(Received April 16, 2018; accepted March 13, 2019)

Keywords: gesture recognition, electronic textile, human-computer interface, biomechanics

Gesture recognition allows distinguishing specific user motions that intend to express a message. The recognized gestures can be used in various applications such as humancomputer interface (HCI), clinical practice including rehabilitation, and personal identification. We propose a method of recognizing upper-limb motion gestures for HCI using electronic textile sensors, which consist of a double-layered structure with complementary resistance characteristics. For gesture recognition, we apply dynamic time warping (DTW) as it exhibits a high performance with simple computations for dynamic signals. We verified the functional feasibility of the proposed method from the data of 10 subjects performing $6 \mathrm{HCI}$ gestures. The gesture classification accuracy for all subjects was $85.4 \%$, although each subject separately achieved a higher performance. In fact, six subjects achieved a perfect recognition performance (100\% recognition accuracy); three subjects achieved an accuracy of $98.6 \%$, and one achieved an accuracy of $97.2 \%$.

\section{Introduction}

Gesture recognition allows to distinguish specific user motions that intend to convey messages and can be used in a myriad of applications such as human-computer interface (HCI) ${ }^{(1-11)}$ medicine applications including rehabilitation, ${ }^{(12-14)}$ and personal identification. ${ }^{(15)}$ Gesture recognition mainly consists of segmenting the acquired signals corresponding to gestures and extracting features that allow gesture classification.

Various kinds of sensors can be used for gesture recognition, with image and depth sensors being the mainstream..$^{(1,2,8,11)}$ For instance, reliable data can be affordably obtained using sensors such as Kinect (Microsoft Co., United States). ${ }^{(2,8)}$ Lahamy and Lichti recognized hand shape and sign language using an approach robust to the user's direction from a depth

*Corresponding author: e-mail: mhryu@jbnu.ac.kr

https://doi.org/10.18494/SAM.2019.2261 
camera. $^{(1)}$ Affordable and small inertial sensors based on MEMS are also widely used for gesture recognition. ${ }^{(3-7,9,15)}$ Kim et al. constructed an inertial measurement unit comprising an accelerometer, a gyroscope, and a magnetometer conforming a data glove to identify the flexion and extension of fingers. ${ }^{(6)}$ Lee et al. recognized gestures corresponding to mouse operation by attaching a similar inertial sensor to the wrist. ${ }^{(7)}$

Electronic textiles (e-textiles) that can be embedded in clothing are being increasingly applied for gesture recognition. ${ }^{(9-15)}$ The electrical properties (e.g., resistance) of flexible e-textiles enable gesture recognition given their variation with bending, stretching, and shape. Moreover, e-textiles provide greater wearing comfort and flexibility than sensors implemented on solid electronics. Bobin et al. constructed a sensor using conductive threads to mount it on the elbow, and the acquired resistance signals were used to identify five levels of elbow flexion and extension with a support vector machine. ${ }^{(9)}$ Gibbs and Asada estimated the angles of knee and hip joints using e-textiles mounted on pants through linear regression. ${ }^{(12)}$ Tognetti et al. fabricated a double-layered sensor by attaching two pieces of e-textile to express a complementary pattern of resistance during flexion and extension. ${ }^{(13)}$ Specifically, the pattern exhibits an increase in resistance for one sensor layer and a decrease in resistance for the other. Then, the angle was estimated using the difference between the signals from the two sensor layers. This sensor conforms a new concept for goniometers, and Santos et al. used a doublelayered goniometer to recognize hand movements for supporting laparoscopic surgery using robots. ${ }^{(14)}$ The authors used rule-based classification to heuristically analyze the sensor patterns for recognition.

In this paper, we propose a hand gesture recognition method for $\mathrm{HCI}$ using e-textiles. The sensor used has a double-layered structure to exhibit the above-mentioned complementary resistance patterns. Although various recognition methods, such as dynamic time warping $(\mathrm{DTW}),{ }^{(2,4)}$ a genetic algorithm, ${ }^{(11)}$ a hidden Markov model, ${ }^{(14)}$ and rule-based classification, ${ }^{(7,13)}$ can be used with this sensor, we selected DTW given its high performance obtained from simple computations on dynamic signals. We validated the functional feasibility of the proposed approach from the data of 10 subjects performing six HCI gestures. ${ }^{(7)}$

\section{Materials and Methods}

\subsection{E-textile sensor and data acquisition}

A conductive e-textile $\left(0.80 \mathrm{~mm}\right.$ thickness, EeonTex ${ }^{\mathrm{TM}}$ NW170-PI-20, Eeonyx Corp., United States) was cut into squares of $20 \times 120 \mathrm{~mm}^{2}$, with the cut along the weft for the shortest side. Two conductive stainless-steel threads $(28 \Omega / \mathrm{ft}$, DEV-11791, Sparkfun Electronics, United States) were sewn $10 \mathrm{~mm}$ from the long end at $2 \mathrm{~mm}$ intervals to connect the wires, as shown in Fig. 1. The stitched parts were heated and pressed using an impulse heat sealer for contact improvement between the e-textile and the conductive thread. We prepared the two sensor layers using this procedure. Then, a double-coated foam tape (cat. \#2240, $24 \mathrm{~mm}$ width, $2 \mathrm{~mm}$ thickness, $3 \mathrm{M}$, United States) was cut according to the length of the sensor size to attach one layer on each side of the tape, resulting in the double-layered sensor (Fig. 1). We prepared three 


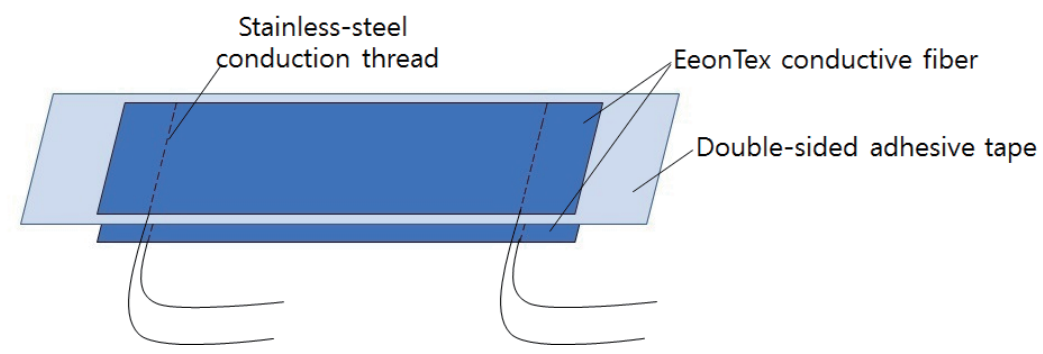

Fig. 1. (Color online) E-textile sensor with double-layered structure.

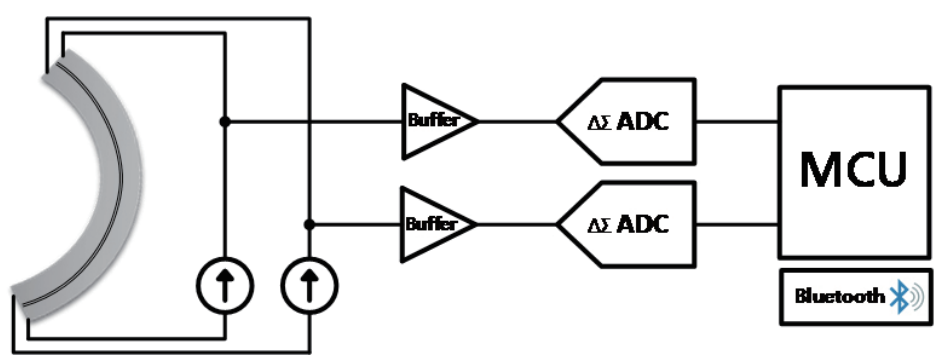

Fig. 2. (Color online) Data acquisition system.

doubled-layered sensors for experiments and attached the sensors to the shoulder (two sensors) and elbow (one sensor) of each participant, as detailed in Sect. 2.3.

The proposed data acquisition system is illustrated in Fig. 2 and consists of the doublelayered e-textile sensor, a constant current source supplying the sensor, a buffer (voltage follower) to obtain a low-impedance sensor output, an analog-to-digital converter (ADC) to obtain digital measurement signals, and a microcontroller for expressing the sensor voltage as resistance and transmitting it to a PC for real-time data acquisition and processing (Fig. 2).

The constant current source allows the resistance variation of the sensor to be converted into corresponding voltage signals. This source was implemented using an adjustable current source IC (LM334, Texas Instruments, Inc., United States). The output resistance of the e-textile sensor can affect the input impedance of the ADC by about $200 \mathrm{k} \Omega$. Hence, we inserted a buffer (TLV2462, Texas Instruments, Inc.) to reduce the impedance at the ADC input for accurate voltage measurements. A 16-bit delta-sigma ADC (ADS1115, Texas Instruments, Inc.) quantized the measured voltage from each sensor to calculate the resistance in the microcontroller, which wirelessly transmitted the resistance signals to a PC through a Bluetooth module at a rate of $50 \mathrm{~Hz}$.

\subsection{Gesture recognition using DTW}

Figure 3 shows the output signal from a measured sensor resistance according to various gestures, where the rows and columns correspond to different gestures and sensor outputs, respectively. The sensor mounting and evaluated gestures are detailed in Sect. 2.3. The output of the first sensor (first column in Fig. 3) shows the difference among patterns for the six gestures. Specifically, in the first row ('up' gesture), the signal increases and then decreases. In 


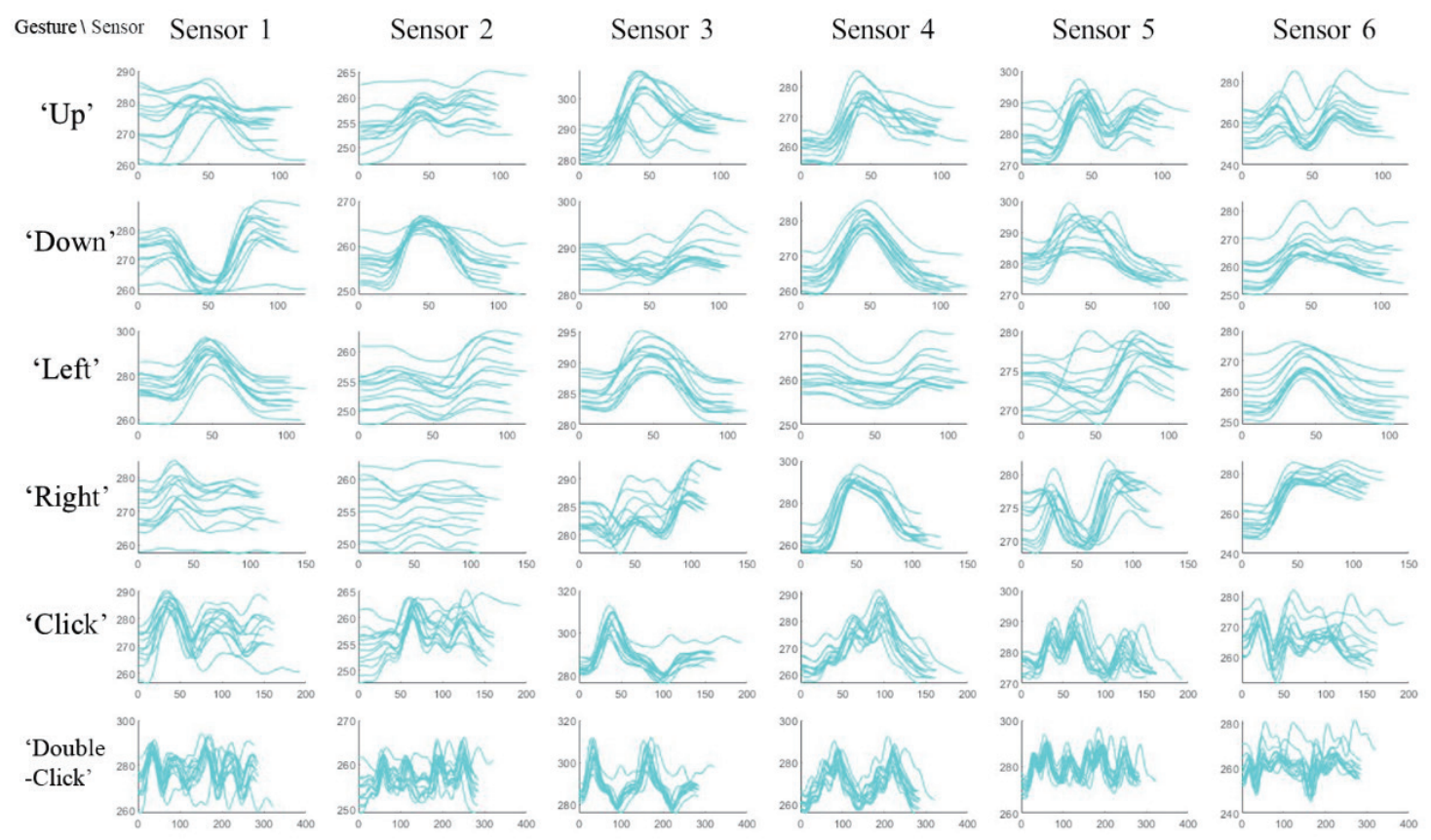

Fig. 3. (Color online) Sensor data (columns) for six gestures (rows). Each pair of columns shows the complementary sensors in the double-layered structure.

the second row ('down' gesture), the signal decreases and then increases, showing an opposite pattern to the up gesture. In the fifth row ('click' gesture), two up and down patterns appear, and in the sixth row ('double-click' gesture), the pattern from the fifth row is repeated twice. Although the third row ('left' gesture) pattern is similar to the first row ('up' gesture) pattern in the first sensor signal, these patterns can be distinguished using data from the second sensor (second column in Fig. 3), suggesting the usefulness of using patterns from multiple complementary sensors.

As mentioned above, the gesture types can be better classified by considering the complementary sensor signals. However, several issues should be addressed. First, the extent of different trials from a gesture can vary. Therefore, the signals should be appropriately scaled for pattern comparison. Likewise, the speed and gesture patterns may vary among subjects and even for the same subject over different executions.

We use DTW to handle these variations of gesture data by warping two time series of data with respect to time. Specifically, two signals are nonlinearly extended or shortened in the time domain, with the warping aiming to minimize the sum of distances between the signals. Then, the sum of distances can be used to quantify the similarity between the two signals at an equal warped data length. Furthermore, DTW is a dynamic programming technique that uses recursive calculations and has been reported to provide a computational efficiency superior to those of other statistical classification techniques. ${ }^{(2,4)}$

To distinguish gestures using DTW, we perform template matching using previously prepared gesture patterns. The motion data to be classified is compared with these patterns to determine the class with the best fitting. Template matching is applied by comparing the 
similarity distances using DTW. The pattern retrieving the smallest sum of distances from DTW is considered as the classification result. Particularly, in this study, the gesture templates are obtained from each subject independently. Therefore, gesture classification results can vary over subjects. Figure 4 shows the gesture patterns used for template matching and generated by averaging multiple time-warped motion data of the same gestures.

\subsection{Experimental protocol}

Three double-layered e-textile sensors were attached at the positions shown in Fig. 5 to efficiently track joint angles during the execution of upper-limb gestures. Specifically, the sensors registered motions from the elbow and shoulder of subjects during flexion and extension, arm raising and falling, and forward and sideways motions. Sensor 1 was mounted such that its center was placed over the olecranon and along the arm length. Sensor 2 was mounted longitudinally at the deltoid-medial center and humerus center so that the shoulder joint laid at the center of the sensor. Sensor 3 was mounted along the dorsal boundary of the posterior deltoid, with one end positioned on the longitudinal centerline of the humerus. The sensors we fabricated did not elongate enough to comply with the joint flexion. ${ }^{(10)}$ Hence, we attached one side of the sensor to the garment using a rubber band.

Ten healthy right-handed subjects (five females and five males) around twenty years old participated in the study. The subjects used their own clothing during the experiments and wore a rash guard with two of the double-layered sensors and an elbow brace with the remaining double-layered sensor. Each subject sequentially performed motions corresponding to six gestures with the right hand, namely, 'up', 'down', 'left', 'right', 'click', and 'double-click'. A

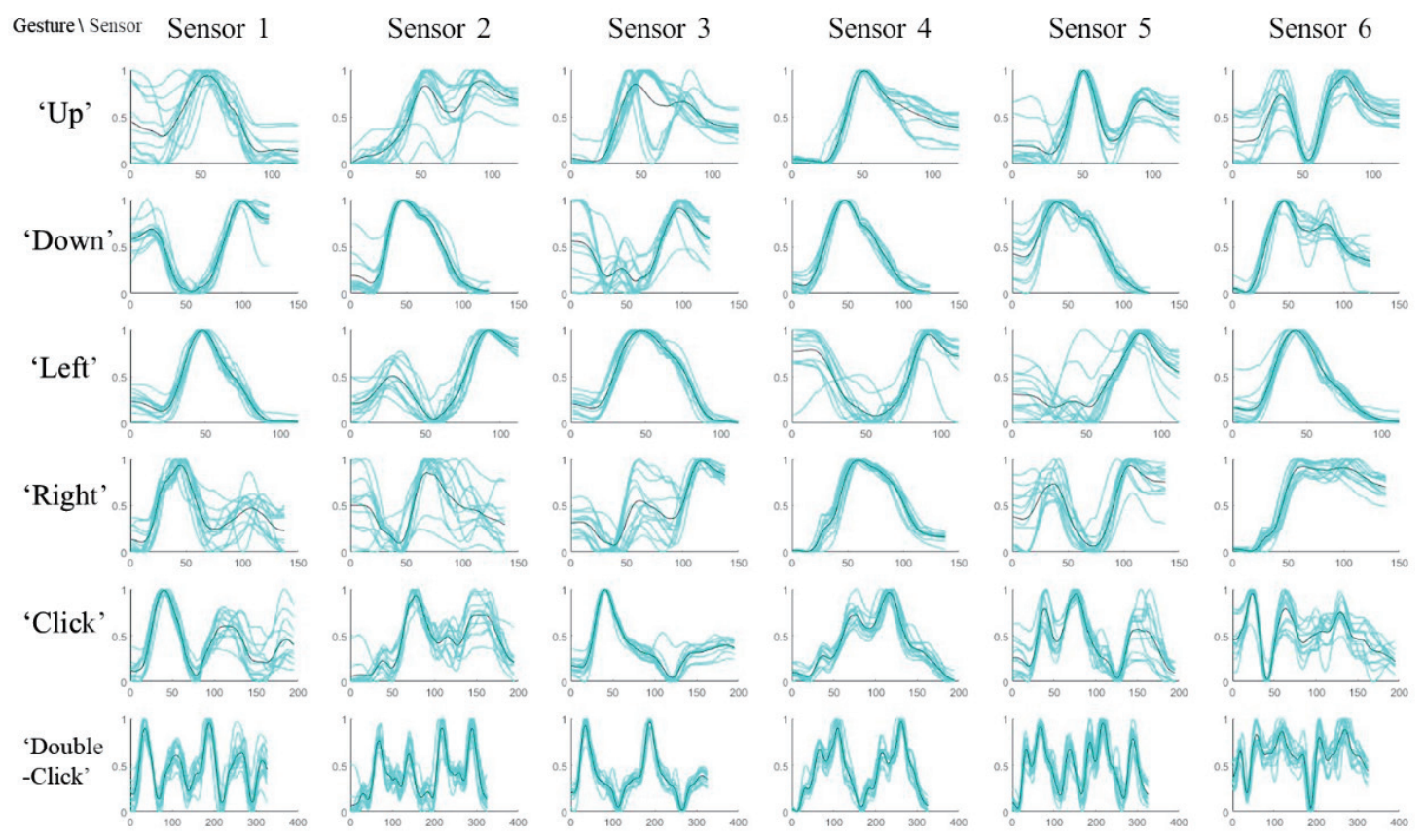

Fig. 4. (Color online) Multiple warped sensor signals (cyan) and gesture patterns (black). 


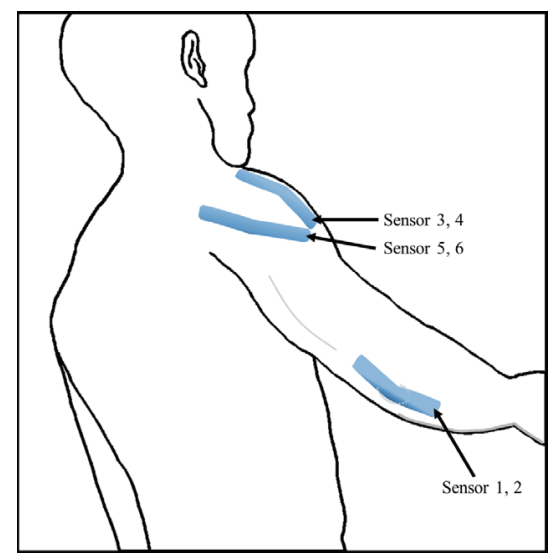

Fig. 5. (Color online) Sensor positioning for gesture measurements.

trial consisted of performing the six gestures twice. Before measurements, we confirmed that every subject understood the experimental procedure and performed the gestures correctly.

Before the first and third trials of experiments, the sensors were calibrated by performing three times the flexion and extension of the forearm joint, the abduction and adduction of the shoulder, and horizontal adduction and horizontal abduction. The sensor positioning was verified at the end of each set of experiments to correct the position of any sensor that might be displaced during gesture execution.

The execution of each gesture took approximately $1.5 \mathrm{~s}$. The subjects grabbed a switch with their right hand and maintained the switch pressed just before the gesture onset until completing the gesture. The switch signal was synchronously stored with the e-textile sensor signals to enable the segmentation of the whole motion sequence into separate gestures. There was a resting period of approximately $1 \mathrm{~s}$ between gestures. Also, there was a longer period of approximately $5 \mathrm{~s}$ before the execution of the second gesture sequence. Each subject performed six experiment trials, and 12 gesture motions were obtained for each of the six gestures.

The resistance time series of the six sensor layers and the switch signal were transmitted to a PC and saved as text files for posterior processing on implementation using MATLAB 9.4 (MathWorks, United States). The gesture motions segmented with the switch signal were identified subjectively considering the sequence of gesture motions. Then, we lowpassfiltered the sensor signals using a 4th-order Butterworth filter with a cutoff frequency of $2 \mathrm{~Hz}$ to suppress information not related to motion and high-frequency noise. In addition, we normalized the six sensor-layer signals from each gesture between zero and one to magnify the signal variation. Then, we employed the DTW function available in MATLAB.

\section{Results and Discussion}

The gesture classification results for the 10 subjects are shown as a confusion matrix in Fig. 6, where the rows and columns correspond to the classified and real gesture classes, respectively. Therefore, the cells along the diagonal correspond to correctly recognized gestures. The additional seventh row shows the true positive rate, or recall (top), and false negative rate (bottom) 
for each gesture class, and the bottom-right cell shows the total accuracy (top) and overall error (bottom).

Gesture 5 ('click') shows the highest accuracy of $95.8 \%$ from the total of 120 executed gestures. Four 'click' gestures were recognized as 'down' gestures and one as 'right' gesture. In contrast, gesture 1 ('up') shows the lowest accuracy of 64.2\%, with 4, 18, 2, and 19 gestures incorrectly recognized as 'down', 'left', 'right', and 'click', respectively. The overall accuracy across subjects and gestures is $85.4 \%$.

Although the classification performance of all the subjects was not satisfactory, the results for each subject show a higher performance, as seen in the classification accuracy listed in Table 1. For six subjects, all the gestures were correctly recognized (100\% accuracy). For three subjects, the accuracy was $98.6 \%$, indicating the misidentification of only one gesture, and the lowest accuracy of $97.2 \%$ was obtained from subject 2 . For subject 2 , whose confusion matrix is shown in Fig. 7, one up gesture was recognized as 'down', and one 'click' gesture was recognized as 'up'.

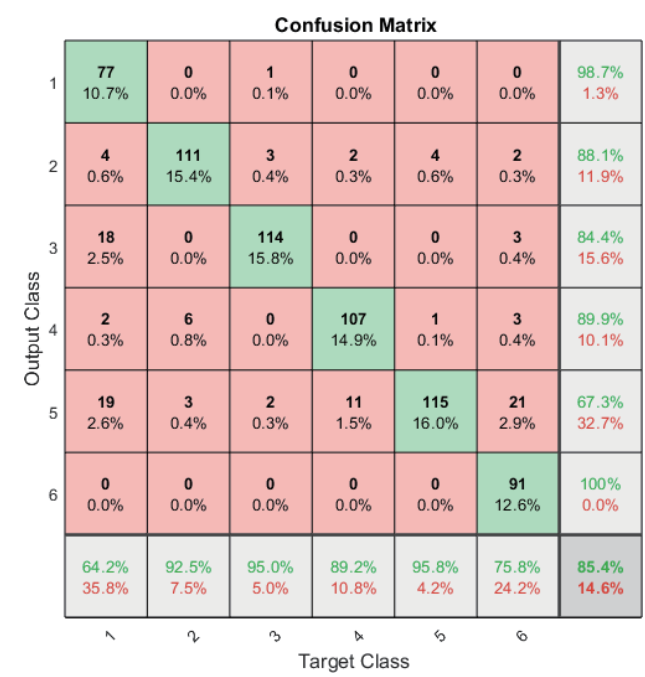

Fig. 6. (Color online) Confusion matrix of general gesture classification.

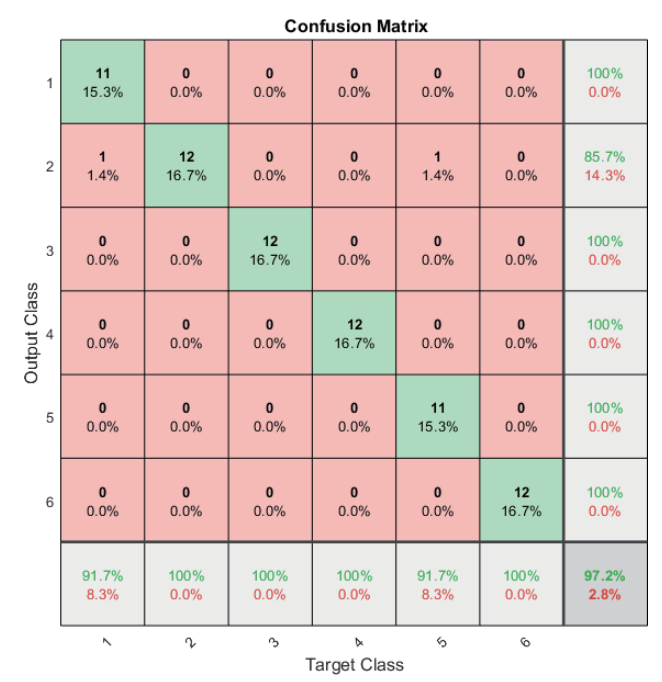

Fig. 7. (Color online) Confusion matrix of gesture classification for subject 2 .

Table 1

Gesture recognition accuracy for each subject.

\begin{tabular}{crrrrrrr}
\hline Subject & Up & Down & Left & Right & Click & DoubleClick & Average \\
\hline 1 & 100.0 & 100.0 & 100.0 & 91.7 & 100.0 & 100.0 & 98.6 \\
2 & 91.7 & 100.0 & 100.0 & 100.0 & 91.7 & 100.0 & 97.2 \\
3 & 100.0 & 100.0 & 100.0 & 100.0 & 100.0 & 100.0 & 100.0 \\
4 & 100.0 & 91.7 & 100.0 & 100.0 & 100.0 & 100.0 & 98.6 \\
5 & 100.0 & 100.0 & 100.0 & 100.0 & 100.0 & 100.0 & 100.0 \\
6 & 100.0 & 100.0 & 100.0 & 100.0 & 100.0 & 100.0 & 100.0 \\
7 & 100.0 & 100.0 & 100.0 & 100.0 & 100.0 & 100.0 & 100.0 \\
8 & 100.0 & 100.0 & 100.0 & 100.0 & 100.0 & 100.0 & 100.0 \\
9 & 100.0 & 100.0 & 100.0 & 91.7 & 100.0 & 100.0 & 98.6 \\
10 & 100.0 & 100.0 & 100.0 & 100.0 & 100.0 & 100.0 & 100.0 \\
\hline Average & 99.2 & 99.2 & 100.0 & 98.3 & 99.2 & 100.0 & 99.3 \\
\hline
\end{tabular}




\section{Conclusions}

We propose a method of recognizing hand gestures for HCI using e-textile sensors resembling a goniometer. Each sensor consists of a double-layered structure with complementary resistance characteristics. For gesture classification, template matching was applied to DTW results, which provide a high performance with inexpensive computations for dynamic signals. The functional feasibility of the proposed method was verified from the data of 10 subjects performing six gestures.

The overall gesture recognition accuracy for all subjects was $85.4 \%$, with the click gesture showing the highest accuracy (95.8\%) and the up gesture the lowest accuracy (64.2\%). As these results may be related to the interindividual variation in anatomical shape, future studies will be devoted to mitigate this variation through a calibration process.

Unlike the overall performance, the accuracy for each subject was high, with six subjects achieving perfect classification (100\% accuracy), whereas gestures from three subjects retrieved 98.6\% accuracy, and those from one subject retrieved 97.2\% accuracy. In future studies, we intend to further improve the method performance by using more sophisticated, albeit complex, classifiers such as statistical classifiers and artificial neural networks.

In this study, the hand gesture recognition method was tried on healthy subjects. However, the recognition of various gestures would be possible based on other joints including finger, knee, and hip joints. Also, the gesture recognition method is expected to be used not only for HCI but also for various applications such as upper limb rehabilitation and gait analysis. For example, it can be used for the daily life support and rehabilitation exercise by classifying daily activities such as drinking and reaching tasks.

\section{Acknowledgments}

This research was supported by the Basic Science Research Program through the National Research Foundation of Korea (NRF), funded by the Ministry of Education (NRF2017R1D1A1B03029739).

\section{References}

1 H. Lahamy and D. D. Lichti: Sensors 12 (2012) 14416. https://doi.org/10.3390/s121114416

2 S. Celebi, A. S. Aydin, T. T. Temiz, and T. Arici: Proc. VISAPP (2013) 620. http://saitcelebi.com/pubs/ visapp2013.pdf

3 Z. Lu, X. Chen, Q. Li, X. Zhang, and P. Zhou: IEEE Trans. Human-Mach. Syst. 44 (2014) 293. https://doi. org/10.1109/THMS.2014.2302794

4 S. Seto, W. Zhang, and Y. Zhou: arXiv preprint (2015) arXiv:1512.06747. https://arxiv.org/abs/1512.06747

5 J. Xu, Y. L. Dong, and Y. Tang: IEEE Control and Decision Conf. (IEEE, 2016) 2763. https://doi.org/10.1109/ CCDC.2016.7531452

6 K. W. Kim, M. S. Lee, B. R. Soon, M. H. Ryu, and J. N. Kim: Technol. Health Care 24 (2016) S223. https://doi. org/10.3233/THC-151078

7 M. S. Lee, K. W. Kim, M. H. Ryu, and J. N. Kim: Sens. Mater. 28 (2016) 655. https://doi.org/10.18494/ SAM.2016.1319

8 F. Milazzo, V. Gentile, A. Gentile, and S. Sorce: Proc. Conf. Complex, Intelligent, and Software Intensive Systems (Springer, 2017) 216. https://doi.org/10.1007/978-3-319-61566-0_21. 
9 M. Bobin, H. Amroun, S. Coquillart, F. Bimbard, and M. Ammi: Proc. IEEE Int. Conf. Systems, Man, and Cybernetics (IEEE, 2016) 2192. https://doi.org/10.1109/SMC.2017.8122934

10 S. H. Han, M. H. Ryu, and J. N. Kim: Sens. Mater. 30 (2018) 1787. https://doi.org/10.18494/SAM.2018.1861

11 R. Kaluri and P. R. CH: Int. J. Eng. Technol. Innovation 8 (2018) 25.

12 P. T. Gibbs and H. Asada: J. Neuroeng. Rehabil. 2 (2005). https://doi.org/10.1186/1743-0003-2-7

13 A. Tognetti, F. Lorussi, G. D. Mura, N. Carbonaro, M. Pacelli, R. Paradiso, and D. De Rossi: J. Neuroeng. Rehabil. 11 (2014). https://doi.org/10.1186/1743-0003-11-56

14 L. Santos, N. Carbonaro, A. Tognetti, J. L. González, E. de la Fuente, J. C. Fraile, and J. Pérez-Turiel: Technologies 6 (2018) 8. https://doi.org/10.3390/technologies6010008

15 C. Nickel and C. Busch: IEEE AES M. 28 (2013) 29.

\section{About the Authors}

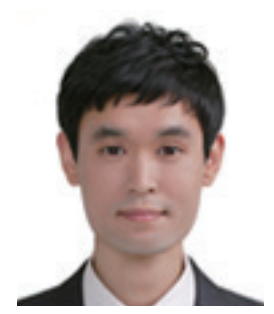

Sang-Ho Han received his B.S. degree in Biomedical Engineering from Chonbuk National University, South Korea, in 2009. From 2010 to 2016, he worked as a researcher at $R \& D$ centers for several companies. He is currently an M.S. student in the Department of Healthcare Engineering at Chonbuk National University. His current research interests are in Internet of Things and embedded systems.

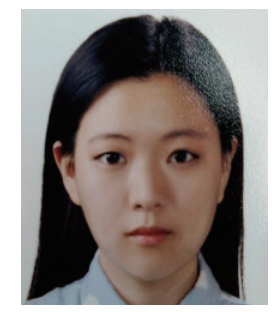

Eun-Ji Ahn received her B.S. degree from Chonbuk National University, South Korea, in 2018. She is currently pursuing her M.S. degree in the Department of Healthcare Engineering, Chonbuk National University, South Korea. Her research interests include biomedical, neural network, electronic, and control engineering.

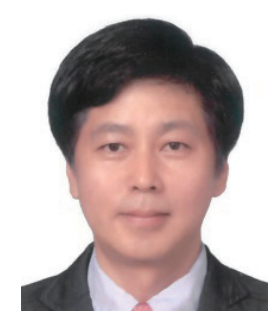

Mun-Ho Ryu received his B.S. degree in Control and Instrumentation Engineering and his Ph.D. degree in Biomedical Engineering from Seoul National University, South Korea, in 1990 and 2004, respectively. He worked for Daewoo Heavy Industry Machine Tool Division from 1990 to 2000. He also worked for Biomedlab Co., Ltd. from 2000 to 2005. He has been a professor at Chonbuk National University since 2005. His research interests are human motion tracking, inertial navigation, telerehabilitation, rehabilitation, and embedded systems.

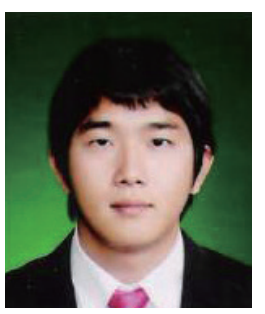

Je-Nam Kim received his B.S. degree in Biomedical Engineering and his M.S. and $\mathrm{Ph} . \mathrm{D}$. degrees in Healthcare Engineering from Chonbuk National University, South Korea, in 2009, 201,1 and 2014, respectively. Since 2014, he has been with CAMTIC Advanced Mechatronics Technology Institute, South Korea. His research interests include embedded software, embedded hardware, and application contents. 